\title{
Multiple resource limitation theory applied to herbivorous consumers: Liebig's minimum rule vs. interactive co-limitation
}

\author{
Erik Sperfeld, ${ }^{1 *}$ Dominik \\ Martin-Creuzburg ${ }^{2}$ and Alexander \\ Wacker' \\ 'Department of Theoretical Aquatic \\ Ecology, Institute of Biochemistry \\ and Biology, University of Potsdam, \\ Am Neuen Palais 10, 14469 Potsdam, \\ Germany \\ ${ }^{2}$ Limnological Institute, University of \\ Constance, Mainaustrasse 252, \\ 78464 Konstanz, Germany \\ *Correspondence: E-mail: \\ eriksperfeld@googlemail.com
}

\begin{abstract}
There is growing consensus that the growth of herbivorous consumers is frequently limited by more than one nutrient simultaneously. This understanding, however, is based primarily on theoretical considerations and the applicability of existing concepts of co-limitation has rarely been tested experimentally. Here, we assessed the suitability of two contrasting concepts of resource limitation, i.e. Liebig's minimum rule and the multiple limitation hypothesis, to describe nutrient-dependent growth responses of a freshwater herbivore (Daphnia magna) in a system with two potentially limiting nutrients (cholesterol and eicosapentaenoic acid). The results indicated that these essential nutrients interact, and do not strictly follow Liebig's minimum rule, which consistently overestimates growth at co-limiting conditions and thus is not applicable to describe multiple nutrient limitation of herbivorous consumers. We infer that the outcome of resource-based modelling approaches assessing herbivore population dynamics strongly depends on the applied concept of co-limitation.
\end{abstract}

\section{Keywords}

Cholesterol, Daphnia magna, eicosapentaenoic acid, essential resources, food quality, herbivore, multi-nutrient limitation, nutritional ecology, von Liebig.

\section{INTRODUCTION}

The availability of essential resources strongly affects the performance of plants and animals. In contrast to primary producers (plants and algae), which acquire essential nutrients mostly as single low-molecular compounds, heterotrophic consumers generally obtain nutrients as packages of complex molecules. Large differences in the elemental and biochemical composition between plants and animals frequently observed in various plant-based communities imply that nutritional constraints should be most pronounced at the plant-herbivore interface (Elser et al. 2000). The predominance of nutritionally imbalanced autotrophic food sources potentially constrains the growth of herbivores and, as a consequence, the efficiency with which energy and essential nutrients are transferred to higher trophic levels.

In addition to elemental nutrients, such as nitrogen $(\mathbb{N})$ and phosphorus (P) (Sterner \& Elser 2002), a number of biochemicals have been shown to be essential dietary compounds, such as certain vitamins (Keating 1985), amino acids (Anderson et al. 2004), polyunsaturated fatty acids (PUFAs; Stanley-Samuelson et al. 1988; Parrish 2009) and sterols (Behmer \& Nes 2003; Martin-Creuzburg \& Von Elert 2009). To identify potentially limiting nutrients and to understand the consequences of nutrient deficiencies is a major challenge of ecological research, particularly in regard to multiple nutrient limitation (co-limitation) of primary (Arrigo 2005; Davidson \& Howarth 2007; Elser et al. 2007; Harpole et al. 2011) and secondary production (Raubenheimer \& Simpson 2004; Martin-Creuzburg et al. 2009; Lukas et al. 2011). For autotrophic organisms, co-limitation scenarios and associated synergistic or antagonistic effects between limiting nutrients, e.g. between $\mathrm{N}$ and $\mathrm{P}$, have been described frequently in a variety of ecosystems (Elser et al. 2007; Allgeier et al. 2011; Harpole et al. 2011). In regard to animal nutrition, however, experimental evidence for such co-limitation scenarios is scarce, although the possibility of a simultaneous limitation by multiple resources has long been recognised (e.g. Tilman 1982). Moreover, little progress has been made to further classify the different types of colimitation for consumers, as has been done for nutrient-limited growth of autotrophic organisms (Saito et al. 2008; Allgeier et al. 2011; Harpole et al. 2011).

Two basic concepts of co-limitation by essential resources have been discussed in the past decades: (1) Liebig's law of the minimum (henceforth Liebig's law), which states that growth is strictly limited by the nutrient in shortest supply relative to demand (Von Liebig 1840), and (2) the multiple limitation hypothesis (henceforth MLH), which states that growth can be limited by more than one nutrient simultaneously (Gleeson \& Tilman 1992). Liebig's law assumes strictly essential resources, i.e. it implies that in a system with multiple limiting nutrients the actually limiting nutrient can change continually and that the switch between limiting nutrients occurs rather abruptly (Fig. 1a). In contrast, the MLH assumes interactive-essential resources, i.e. it considers interactions between limiting nutrients and predicts smooth transitions from a limitation by one nutrient to a limitation by another (Fig. 1b). According to the MLH, all nutrients limit growth to some extent but the strength of a limitation by a particular nutrient depends on the supply relative to the demand. These two concepts of colimitation lead to divergent predictions, as indicated by different shapes of resource-dependent growth isoclines (Fig. 1), especially at colimiting conditions (Tilman 1982). At low resource availabilities, isoclines representing very low growth rates are similar in shape for both the MLH and Liebig's law (Fig. 1a,b). At high resource availabilities, isoclines representing high growth rates differ unambiguously in shape between the two concepts, but the absolute differences in predicted growth rates are marginal (Fig. 2a). However, at intermediate resource availabilities, where growth is strongly colimited, differences in growth rates predicted by the two contradicting concepts are considerable and should not be neglected (Fig. 2b). In this range of resource availabilities, growth rates predicted by the MLH are constrained by more than one resource simultaneously and are subject to interactive effects between co-limiting resources. Consequently, at 
(a)
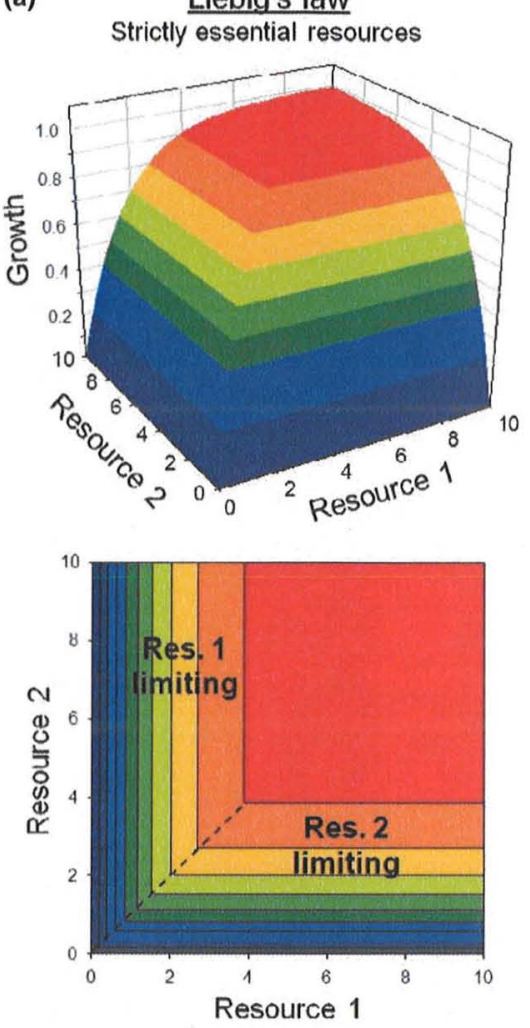

(b)
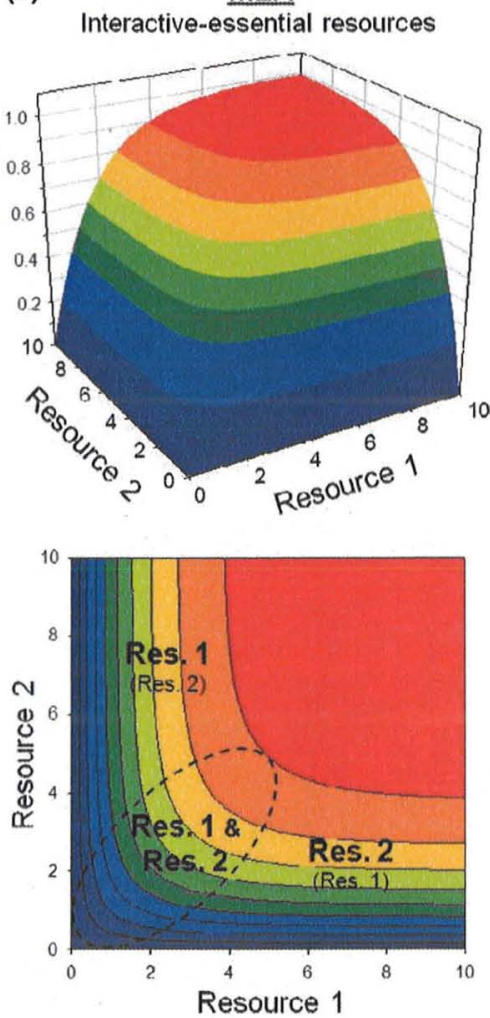

Figure 1 Two concepts of co-limitation for essential resources: (a) Liebig's law of the minimum assuming strictly essential resources (left panels, Bertalanffy model: Table 1, eqn 2, $g_{\max }=1, b 1=b 2=0.6, R 1_{0}=R 2_{0}=0$ ) and (b) the multiple limitation hypothesis (MLH) assuming interactive-essential resources (right panels, Bertalanffy model: Table 1, eqn $6, g_{\max }=1, b 1=b 2=0.6, R 1_{0}=R 2_{0}=0$ ). Resource-dependent growth isoclines (solid lines) indicate equal growth at changing resource availabilities. Liebig's law shows right angle corners of growth isoclines, indicating that growth is strictly limited by only one resource at a certain resource availability. The MLH shows rounded corners of growth isoclines, indicating a smooth transition of limitation by one to the other resource and a range of resource availabilities at which both resources strongly limit growth simultaneously. Resource limitation scenarios are separated by the dashed line.

co-limiting conditions, growth rates predicted by the MLH are lower than those predicted by applying Liebig's law, i.e. by assuming strictly essential resources (Fig. 2a). Thus, the strength of the simultaneous limitation can be assessed by calculating differences in growth rates predicted by the two concepts of co-limitation (Fig. 2b,c).

To understand and accurately predict demographic changes and population dynamics of consumers it is important to know (1) the range of concentrations over which nutrients are co-limiting, (2) the concept of co-limitation which is most applicable and (3) the strength of potential interactive effects mediated by simultaneously limiting nutrients. In a previous study, using the freshwater herbivore Daphnia magna as model organism, we provided experimental evidence for a colimitation of a consumer by sterols and PUFAs (Martin-Creuzburg et al. 2009), but the concept of co-limitation and the magnitude of potential interactive effects remained unclear. Here, we designed an experiment which allows to specifically test the two concepts of co-limitation, i.e. to classify whether two potentially limiting nutrients are either strictly or interactively essential, and to assess the range of nutrient concentrations at which potential interactions may arise, i.e. where growth is limited simultaneously by both nutrients. For this purpose, we used a fullfactorial approach in which the availability of both nutrients, i.e. sterols and PUFAs, was experimentally modified by supplementing increasing amounts of cholesterol and/or eicosapentaenoic acid (EPA) to a steroland PUFA-free diet. We fitted various mathematical models based on the two contradicting concepts of co-limitation to the obtained nutrientlimited growth responses of D. magna and compared the predictive power of these models. Differences in growth rates predicted by the two concepts were used to test our hypothesis that growth rates at co-limiting conditions are overestimated by applying Liebig's minimum rule.

\section{MATERIAL AND METHODS}

\section{Cultivation of organisms and preparation of food}

Stock cultures of the herbivorous crustacean D. magna were maintained for several generations in filtered lake water $(0.2 \mu \mathrm{m}$ pore-sized membrane filter) at $20{ }^{\circ} \mathrm{C}$ and fed ad libitum with the green alga Scenedesmus obliquus (SAG 276-3a, culture collection of algae, University of Göttingen, Göttingen, Germany). For growth experiments, the well ingestible, non-toxic, and sterol- and PUFA-free cyanobacterium Synechococcus elongatus (SAG 89.79) was used as food for D. magna (Martin-Creuzburg et al. 2008). S. elongatus and S. obliquus were cultured semi-continuously (dilution rates: $0.2 \mathrm{~d}^{-1}$ and $0.4 \mathrm{~d}^{-1}$, respectively) at a light : dark cycle of $16: 8 \mathrm{~h}$ in aerated $2 \mathrm{~L}$ flasks using WC medium with vitamins (Guillard 1975) and illumination at $40 \mu \mathrm{mol} \mathrm{m} \mathrm{m}^{-2} \mathrm{~s}^{-1}$ and $120 \mu \mathrm{mol} \mathrm{m} \mathrm{m}^{-2} \mathrm{~s}^{-1}$, respectively. Prior to the experiment, a serial dilution of the $S$. elongatus culture was used to establish a carbon - light extinction regression equation. Therefore, 
(a)
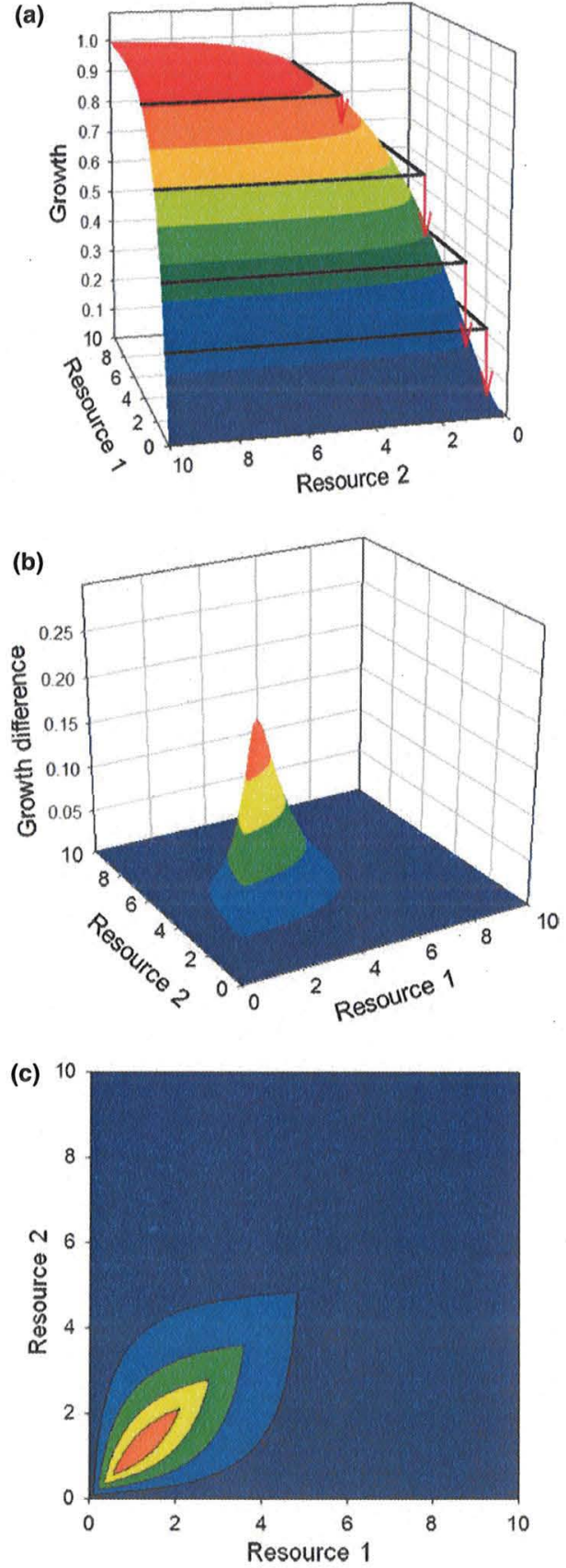

Figure 2 (a) Depiction of the calculation of differences in growth rates predicted by a model based on the multiple limitation hypothesis (MLH) and a model based on Liebig's law (for models and parameters used see Fig. 1). This calculation method assesses the strength of the simultaneous limitation by both resources (but see Appendix S3). The coloured surface indicates the model based on the MLH and the black lines indicate growth isoclines of the model based on Liebig's law. Red arrows indicate examples of calculated growth rate differences (b) \& (c) Calculated differences in growth rates between the contradicting colimitation models, indicating the strength of the simultaneous limitation mediated by interactive effects between co-limiting resources. The simultaneous limitation by both resources is most pronounced at strongly co-limiting conditions (interactive range, within the light blue ellipse). In this interactive range growth is lower than predicted by assuming strictly essential resources according to Liebig's law (see also Appendix S3). Note the change of direction of the $x$-axis from (a) to (b) \& (c). aliquots of each dilution step were measured for photometric light extinction $(800 \mathrm{~nm})$, filtered onto pre-combusted glass-fibre filters (Whatman GF/F, $25 \mathrm{~mm}$ diameter, Schleicher \& Schuell, Dassel, Germany) and, after drying for 2 days at $50^{\circ} \mathrm{C}$, analysed for particulate organic carbon using a carbon analyser (HighTOC $+\mathrm{N}$, Elementar). During the experiment, the obtained carbon - light extinction equation was used to calculate the carbon concentration of the daily new prepared food suspensions (see below).

Cholesterol- and EPA-containing liposomes used as food supplements were prepared according to Martin-Creuzburg et al. (2008). The produced liposome suspensions were stored at $-20^{\circ} \mathrm{C}$ until experimental food treatments were prepared. During the experiment, the concentration of cholesterol and EPA in liposome stock suspensions was determined repeatedly by gas chromatography as described in Wacker \& Martin-Creuzburg (2007). One microlitre of the liposome suspensions contained either $0.36 \pm 0.05 \mu \mathrm{g}$ cholesterol (mean $\pm \mathrm{SD}$, $n=10)$ or $0.17 \pm 0.04 \mu \mathrm{g}$ EPA $(n=7)$; these values were related to the total carbon content of the food suspensions (provided by both $S$. elongatus and liposomes) to determine dietary cholesterol and EPA concentrations (for details see Appendix S1).

\section{Growth Experiment}

Fourth-clutch juveniles of $D$. magna, hatched within $12 \mathrm{~h}$ in filtered lake water containing $2 \mathrm{mg} \mathrm{C} \mathrm{L}^{-1}$ of $S$. elongatus as food, were used for the growth experiment, which was conducted at $20^{\circ} \mathrm{C}$. Initial dry mass of animals $\left(D M_{0}\right)$ was determined from three subsamples of 15 juveniles. Six juveniles were transferted to glass beakers filled with $200 \mathrm{~mL}$. filtered lake water and provided with saturating food concentrations of $S$. elongatus $\left(2 \mathrm{mg} \mathrm{C} \mathrm{L}^{-1}\right)$. Supply gradients of cholesterol and EPA were generated by supplementing increasing amounts of cholesterol- and EPA-containing liposomes to the experimental beakers. Eight supply levels of cholesterol were prepared by adding $0,5,7,9,11,13,16$ and $20 \mu \mathrm{L}$ of cholesterol-containing liposomes to the beakers, resulting in dietary cholesterol concentrations of $0-17.6 \mu \mathrm{g} \mathrm{mg} \mathrm{C}^{-1}$ (see Appendix S1). Similarly, for each cholesterol supply level, eight EPA supply levels were prepared by adding $0,1,2,4,7,11,16$ and $22 \mu \mathrm{L}$ of EPA-containing liposomes, resulting in dietary EPA concentrations of $0-9.0 \mu \mathrm{g} \mathrm{mg} \mathrm{C} \mathrm{C}^{-1}$. In this way, we created 64 dietary treatments $(8 \times 8$ supply levels $)$ differing in their cholesterol and EPA availability (see Appendix S1). Each food treatment was duplicated resulting in 128 experimental beakers. Previous supplementation experiments revealed that the variability around treatment means is low in this kind of experimental set-up (Martin-Creuzburg et al. 2009, 2010). Therefore, food treatments were replicated only twice to allow for a high number of nutrient supply levels needed for reliable regression analyses.

The animals were transferred daily to beakers containing renewed food suspensions until the end of the 5.5 days lasting growth experiment. At the end of the experiment, individuals of each replicate were transferred into pre-weighed aluminium boats and weighed using an electronic balance ( $\pm 1 \mu \mathrm{g}, \mathrm{CP} 2 \mathrm{P}$; Sartorius, Goettingen, Germany) after drying for $48 \mathrm{~h}$ at $50^{\circ} \mathrm{C}$ to determine the final dry mass of animals $\left(D M_{t}\right)$. Mass-specific growth rates, $g\left(\mathrm{~d}^{-1}\right)$, of $D$. magna were determined as the increase in dry mass from the beginning $\left(D M_{0}\right)$ to the end of the experiment $\left(D M_{t}\right)$ using the equation

$g=\left(\ln D M_{t}-\ln D M_{0}\right) t^{-1}$,

where $t$ is the duration of the experiment in days. 


\section{Statistical analyses}

Nutrient-limited growth responses of consumers can be described by saturation functions, similar to a Holling's Type II functional response (Holling 1959). The increase in growth rate $(g)$ in response to increasing resource concentration $(R)$, approaching the asymptotic maximum growth rate $\left(g_{\max }\right)$, can be mathematically expressed using the Monod function (Monod 1950) with $k$ as half saturation constant:

$g=g_{\max } \frac{R}{k+R}$

or the Bertalanffy function (Von Bertalanffy 1957) with $b$ as Bertalanffy growth coefficient:

$g=g_{\max }\left(1-e^{-b R}\right)$.

Both functions have been used previously to describe nutrientlimited growth of Daphnia (e.g. Müller-Navarra et al. 2000; Wacker \& Von Elert 2001; Martin-Creuzburg et al. 2009). Based on these functions that can be used in case of a single limiting nutrient, we used different co-limitation models to describe the dependency of growth rates on two nutrients by adding an additional Monod (Table 1, eqn 1 \& 3, see also Saito et al. 2008) or Bertalanffy function (Table 1, eqn 2 $\&$ 6, see also Baule 1918) for the second nutrient. The co-limitation concept of Liebig's law (Fig. 1a) is expressed as the minimum of one of the two Monod (Table 1, eqn 1) or Bertalanffy (eqn 2) functions. The co-limitation concept based on the MLH (Fig. 1b) is described by the product of two Monod or Bertalanffy functions (Table 1, eqn 3 \& 6) and, for comparison, by two models assuming nutrient arrival according to a Poisson process, i.e. the Poission arrival time model (PAT, Table 1, eqn 4) developed by O'Neill et al. (1989) and a model variant of the synthesising unit concept (SU, Table 1, eqn 5) originally developed by Kooijman (1998). The SU model presented here assumes a homogeneous environment where nutrient concentrations can be substituted for nutrient fluxes (Muller et al. 2001).

The functions representing different concepts of co-limitation (Table 1) were fitted to the mass-specific growth rates of $D$. magna using the nonlinear least-squares procedure of the statistical software package R v. 2.5.1. The Gauss-Newton algorithm was applied with a tolerance for convergence of 0.0001. Initial starting values for parameters were estimated based on previous work (MartinCreuzburg et al. 2009; Lukas et al. 2011; Sperfeld \& Wacker 2011). To test for local minima after successful fitting procedures, cach model was fitted again several times to the data using different combinations of starting values. All possible combinations of the (five) fitted parameters, the fitted parameters $-15 \%$ and the fitted parameters $+15 \%$ were used as starting values resulting in $3^{5}=243$ fitting procedures with different starting values per model. The fitted parameter set resulting in the lowest negative log-likelihood $(N L L=-2 \cdot \log L)$ was chosen, because a lower $N L L$-value as goodness of fit measure indicates a model which is more likely to be correct. To compare different co-limitation models, differences between the $N L L$-values $(\triangle N L L)$ of two models were used to calculate the probability $(\mathrm{P})$ that the 'worse model' (higher $N L L$ ) was more likely to be correct than the "better model' (lower $N L L$ ) (modified after Motulsky \& Cristopoulus 2004):

$\mathrm{P}=\frac{\exp (-0.5 \cdot \Delta N L L)}{1+\exp (-0.5 \cdot \Delta N L L)}$.

For the purpose of comparison, we also applied additional colimitation models to our growth rate data which showed equal or worse goodness of fit measures than the presented models (see Appendix S2).

To assess the strength of the simultaneous growth limitation, potentially mediated by interactive effects between cholesterol and EPA, we calculated the differences of growth predicted by a model based on the MLH and a model based on Liebig's law (Fig. 2a). At a given resource availability, we subtracted growth rates predicted by the parameters of the fitted MLH model from growth rates predicted by a minimum function using the same parameters as the MLH model (Fig. 2, see Appendix S3 for more details). For comparison, we also calculated the differences in predicted growth rates using the estimated parameters both for the model based on the MLH and the model based on Liebig's law (i.e. different parameter sets for the two different co-limitation models, see Appendix S3).

Table 1 Co-limitation models based on the concept of Liebig's law assuming strictly essential resources (eqn 1 \& 2) and the MLH assuming interactive-essential resources (eqn 3-6). Growth rates (g) were described using models based on Monod functions (Saito et al. 2008), Poisson arrival time (PAT, O'Neill et al. 1989), synthesising units (SU, Muller et al. 2001), or Bertalanffy growth functions (Von Bertalanffy 1957; but see also Baule 1918). More information and additional co-limitation models can be found in Appendix S2

\begin{tabular}{|c|c|c|}
\hline Model & Eqn & Function \\
\hline Liebig's law (strictly essential resources) & & {$\left[R 1^{x}\right.$} \\
\hline Monod & (1) & $g=\min \left[g_{\max } \frac{R 1^{*}}{k 1+R 1^{x}}, g_{\max } \frac{R L^{x}}{k 2+R 2^{x}}\right]$ \\
\hline Bertalanffy & (2) & $g=\min \left[g_{\max }\left(1-e^{-\not 1 R 1^{x}}\right), g_{\max }\left(1-e^{-122 R 2^{X}}\right)\right]$ \\
\hline MLH (interactive-essential resources) & & $R 1^{x} \quad R 2^{x}$ \\
\hline Monod & (3) & $g=g_{\max } \overline{k 1+R 1^{x}} \cdot \overline{k 2+R 2^{x}}$ \\
\hline PAT & (4) & $g=\frac{g_{\max } R 1^{x} R 2^{x}\left(k 2 R 1^{x}+k 1 R 2^{x}\right)}{R 1^{x} R 2^{x}\left(k 2 R 1^{x}+k 1 R 2^{x}\right)+\left(k 2 R 1^{x}\right)^{2}+k 1 k 2 R 1^{x} R 2^{x}+\left(k 1 R 2^{x}\right)^{2}}$ \\
\hline SU & (5) & $g=1 / \frac{1}{g_{\max }}+\frac{k 1}{g_{\max } R 1^{x}}+\frac{k 2}{g_{\max } R 2^{x}}-\left(\frac{g_{\max } R 1^{x}}{k 1}+\frac{g_{\max } R 2^{x}}{k 2}\right)^{-1}$ \\
\hline Bertalanffy & (6) & $g=g_{\max }\left(1-e^{-b 1 R 1^{X}}\right) \cdot\left(1-e^{-\not 2 R 2^{X}}\right)$ \\
\hline
\end{tabular}

The minimum function ( $\mathrm{min}$ ) specifies $g$ as the lower value of the two terms in parentheses. Description of parameters: $g_{\text {max }}$, asymptotic maximum growth rate; $k 1$ and $k 2$, Monod half saturation constants, $b 1$ and $b 2$, Bertalanffy growth coefficients for resources $R 1$ and $R 2$, respectively; $R 1^{* x}=R 1-R 1_{0}$ and $R 2^{x}=R 2-R 2_{0}$ with $R 1_{0}$ and $R 2_{0}$ as threshold resource concentrations for zero growth. 

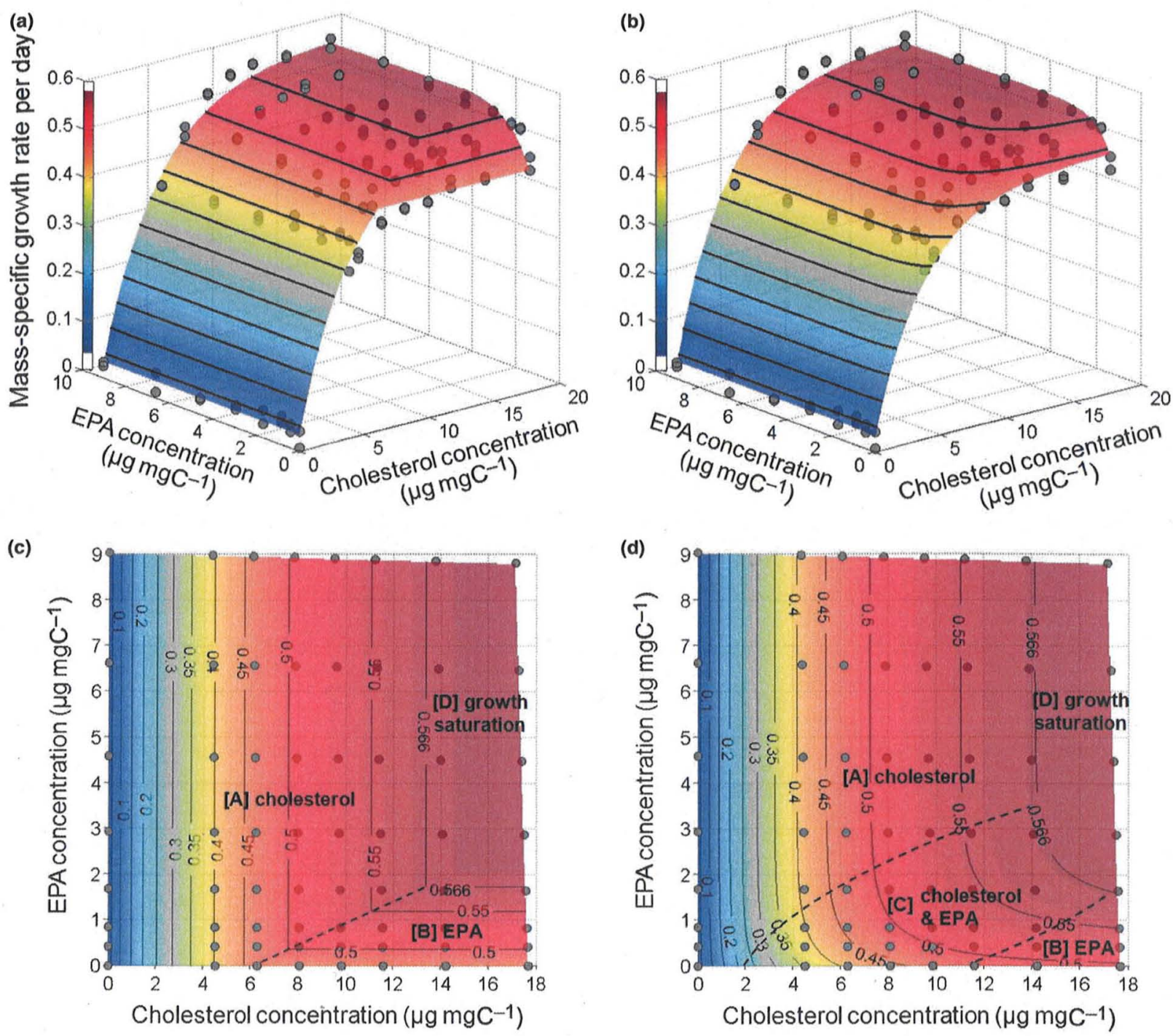

Figure 3 Mass-specific growth rates of Daphnia magna (points) depending on dietary cholesterol and eicosapentaenoic acid (EPA) concentrations. The coloured surfaces represent nonlinear least square fits through growth rates for the Bertalanffy model based on (a \& c) Liebig's law (Table 1, eqn 2; Table 2, model II) and (b \& d) the multiple limitation hypothesis (MLH) (Table 1, eqn 6; Table 2, model VI). Solid lines on the model surface in the three-dimensional plots (a \& b) denote resource-dependent growth isoclines with their respective growth rates given in the two-dimensional plots (c \& d). The dashed line in panel (c) separates ranges of resource availabilities at which growth rates were limited by only one nutrient, i.e. either $[A]$ cholesterol or $[B]$ EPA. The dashed line in panel (d) separates ranges of resource availabilities at which growth rates were limited predominantly either by $[\mathrm{A}]$ cholesterol or $[\mathrm{B}]$ EPA or $[\mathrm{C}]$ by both nutrients simultaneously. In both cases there is a range of resource availabilities $[\mathrm{D}]$ at which growth rates were saturated $(g \geq 0.566)$, i.e. neither cholesterol nor EPA was limiting.

\section{RESULTS}

Without cholesterol supply, the growth of D. magna was very poor, irrespective of EPA supply (mean $\pm \mathrm{SD}, g=0.037 \pm 0.015, n=16$, Appendix S4). This pronounced growth limitation was offset primarily by increasing cholesterol availability (Fig. 3a,b, see also Appendix S4). With increasing cholesterol availability, growth rates were also affected by the availability of dietary EPA (Fig. 3a,b, Appendix S4). The cholesterol-dependent growth responses at each EPA supply level showed saturation curves. The EPA-dependent growth responses showed saturation curves only when at least medium amounts of cholesterol ( $\geq 6 \mu \mathrm{g} \mathrm{mgC}^{-1}$ ) were supplied (Fig. 3a,b, Appendix S4). Maximum growth rates (mean $\pm \mathrm{SD}, g=0.566 \pm 0.013, n=18$ ) were observed at high dietary cholesterol $\left(\geq 13.7 \mu \mathrm{g} \mathrm{mgC}^{-1}\right)$ and high dietary EPA $\left(\geq 2.8 \mu \mathrm{g} \mathrm{mgC}^{-1}\right)$ concentrations.

First, we applied co-limitation models based on Liebig's law to describe the growth of $D$. magna depending on dietary cholesterol and EPA concentrations by fitting minimum functions to growth rates (Table 1, eqn 1 \& 2). Models based on both Monod functions (Table 2, model I) and Bertalanffy functions (Table 2, model II) described the growth responses of $D$. magna very well, which was indicated by highly significant model parameters and high adjusted $R^{2}$-values (Table 2). The model based on Bertalanffy functions was used for presentation (Fig. 3a) because it resulted in a substantially better fit than the model based on Monod functions $(\Delta N L L=44.3$, $P<0.0001)$. Contrasting the models based on Liebig's law with their 
Table 2 Results of nonlinear least square fits through mass-specific growth rates, $g\left(\mathrm{~d}^{-1}\right)$ of Daplsnia magna $(n=128)$ with carbon-based cholesterol $(R 1)$ and EPA $(R 2)$ concentration as independent variables. Equations (eqn) and parameters $\left(g_{\text {nax }}, k 1, b 1, k 2, b 2, R 1_{0}, R 2_{0}\right)$ are specificd in Table 1 . Fitting results of additional co-limitation models are given in Appendix S2

\begin{tabular}{|c|c|c|c|c|c|c|}
\hline & \multicolumn{2}{|c|}{ Liebig's law (strictly essential resources) } & \multicolumn{4}{|c|}{ MLH (interactive-essential resources) } \\
\hline & Monod & Bertalanffy & Monod & PAT & SU & Bertalanffy \\
\hline Model & I & II & III & IV & V & VI \\
\hline Eqn & (1) & (2) & (3) & (4) & (5) & (6) \\
\hline$N L L$ & -607.5 & -651.8 & -613.5 & -626.6 & -626.6 & -663.4 \\
\hline Adj. $R^{2}$ & 0.980 & 0.986 & 0.981 & 0.983 & 0.983 & 0.987 \\
\hline$g_{\max } \pm \mathrm{SE}$ & $0.745 \pm 0.016$ & $0.588 \pm 0.005$ & $0.688 \pm 0.011$ & $0.710 \pm 0.013$ & $0.710 \pm 0.013$ & $0.578 \pm 0.004$ \\
\hline$t$-value & $(45.8)^{* * *}$ & $(114.5)^{* * *}$ & $(63.6)^{* * *}$ & $(56.5)^{* * *}$ & $(56.6) * * *$ & $(142.0)^{* * *}$ \\
\hline$k 1$ or $b 1 \pm \mathrm{SE}$ & $3.94 \pm 0.27$ & $0.24 \pm 0.01$ & $2.76 \pm 0.17$ & $3.23 \pm 0.20$ & $3.23 \pm 0.20$ & $0.27 \pm 0.01$ \\
\hline$t$-value & $(14.5)^{* * *}$ & $(32.7)^{* * *}$ & $(16.3)^{* * *}$ & $(16.5)^{* * *}$ & $(16.5)^{* * *}$ & $(37.3)^{* * *}$ \\
\hline$k 2$ or $b 2 \pm \mathrm{SE}$ & $2.11 \pm 0.40$ & $1.03 \pm 0.14$ & $0.07 \pm 0.02$ & $0.31 \pm 0.08$ & $0.31 \pm 0.08$ & $1.51 \pm 0.24$ \\
\hline$t$-value & $(5.2)^{* * *}$ & $(7.3)^{* * *}$ & $(3.3)^{* *}$ & $(4.2)^{* * *}$ & $(4.2)^{* * *}$ & $(6.2)^{* * *}$ \\
\hline$R 1_{0} \pm \mathrm{SE}$ & $-0.20 \pm 0.04$ & $-0.25 \pm 0.04$ & $-0.16 \pm 0.03$ & $-0.17 \pm 0.03$ & $-0.17 \pm 0.03$ & $-0.24 \pm 0.03$ \\
\hline$t$-value & $(-5.4)^{* * *}$ & $(-6.5)^{* * *}$ & $(-5.6)^{* * *}$ & $(-5.8)^{* * *}$ & $(-5.8)^{* * *}$ & $(-6.9)^{* * *}$ \\
\hline$R 2_{0} \pm \mathrm{SE}$ & $-3.86 \pm 0.68$ & $-1.47 \pm 0.21$ & $-0.39 \pm 0.12$ & $-0.75 \pm 0.18$ & $-0.75 \pm 0.18$ & $-1.28 \pm 0.22$ \\
\hline$t$-value & $(-5.7)^{* * *}$ & $(-7.0)^{* * *}$ & $(-3.4)^{* *}$ & $(-4.3)^{* * *}$ & $(-4.3)^{* * *}$ & $(-5.8)^{* * *}$ \\
\hline
\end{tabular}

For the nonlinear fits (d.f. $=123)$ the adjusted $R^{2}$ (adj. $\left.R^{2}\right)$ and the negative $\log$-likelihood $(N L L=-2 \cdot \log L)$ are denoted; parameters \pm standard error $($ SE) are given and $t$-values are provided in parentheses together with corresponding significance levels $(* * P<0.01, * * * P<0.001)$.

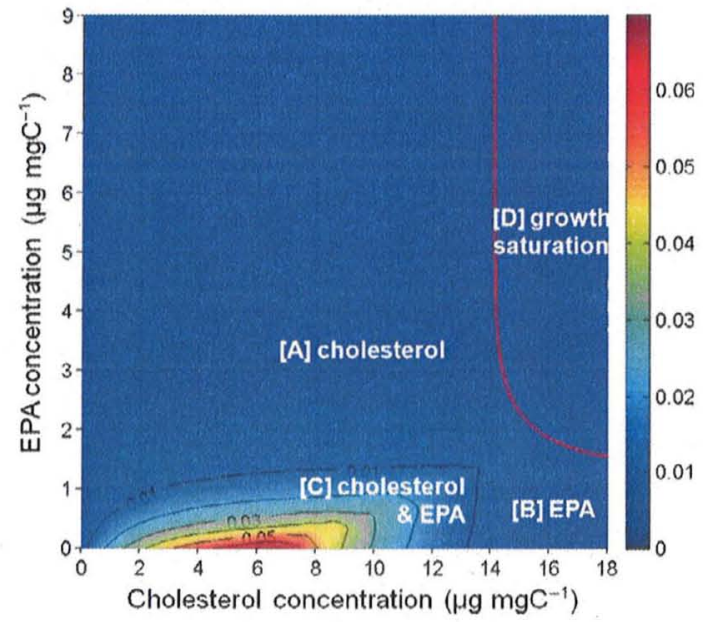

Figure 4 Strength of the simultaneous growth limitation of Daphnia magna mediated by interactive effects between cholesterol and EPA calculated as the difference in predicted growth rates $\left(\mathrm{d}^{-1}\right)$ between a model based on the multiple limitation hypothesis (MLH) (Table 1, eqn 6) and a model based on Liebig's law (Table 1, eqn 2) using the same parameter set (Table 2, model VI). The isocline $=0.01$ frames the interactive range where growth was limited simultaneously by $[\mathrm{C}]$ cholesterol and EPA. The blue area indicates ranges of resource availabilities where either $[\mathrm{A}]$ cholesterol or $[\mathrm{B}] \mathrm{EPA}$ was the dominant limiting nutrient, or [D] neither cholesterol nor EPA limited growth, i.e. growth saturation (separated by the red solid line, $g=0.566$, see also Fig. $3 d$ ).

MLH counterparts indicated that the MLH models more accurately described the nutrient-limited growth responses than the Liebig based approaches, irrespective of whether the models were based on Monod functions (Table 2, model I \& III, $\triangle N L L=5.95, P=0.049$ ) or on Bertalanffy functions (Table 2, model II \& VI, $\Delta N L L=11.6$, $P=0.003)$. Likewise, for the MLH, the model based on Bertalanffy functions (Table 2, model VI, Fig. 3b) resulted in a much better fit than the model based on Monod functions (Table 2, model III, $\triangle N L L=49.9, P<0.0001)$. The PAT model (Table 2, model IV) and the SU model (Table 2, model V) resulted in the same parameter values and goodness of fit measures. These models fitted the data better than the Monod model based on either the MLH (Table 2, model III, $\triangle N L L=13.1, P=0.001$ ) or on Liebig's law (Table 2, model I, $\Delta N L L=19.0, P=0.0001)$, but worse than the Bertalanffy model based on either the MLH (Table 2, model VI, $\Delta N L L=36.8$, $P<0.0001$ ) or on Liebig's law (Table 2, model II, $\Delta N L L=25.3$, $P<0.0001)$. In summary, this means that although the Bertalanffy models described growth generally better than all other models based on hyperbolic functions, the concept of the MLH described growth better than Liebig's law across the different model types.

According to Liebig's law, ranges of resource availabilities at which either cholesterol or EPA limited the growth of $D$. magna were separated by the intersecting line through the right angles of growth isoclines (Fig. 3c). The MLH suggested three ranges of nutrient availabilities corresponding to different limitation scenarios (Fig. 3d) where growth is limited primarily by the availability of (1) cholesterol, (2) EPA or (3) by the availability of both nutrients simultaneously, which results in a curved shape of the nutrient-dependent growth isoclines. The existence of a nutrient range where growth is limited by both nutrients simultaneously according to the MLH contradicts Liebig's law. The simultaneous limitation of growth by cholesterol and EPA was also verified by comparing actual growth rate responses within the nutrient range of co-limiting conditions (see Appendix S5).

To assess the strength of the interactive effects mediated by the two co-limiting nutrients (Fig. 4) we calculated the differences in growth rates predicted by the Bertalanffy model based on the MLH (Table 1 , eqn 6, Table 2, model VI) and the Bertalanffy model based on Liebig's law (Table 1, eqn 2) using the same parameter values (Table 2, model VI). With this approach, the range of nutrient availabilities at which growth is limited either by cholesterol or EPA can be more accurately assessed than by simply comparing growth isoclines (cf. Figs $3 \mathrm{~d}$ and 4). The simultaneous limitation by both nutrients is predicted at low to medium cholesterol (1-11 $\mathrm{g} \mathrm{mgC}^{-1}$ ) and low EPA supply

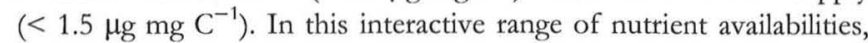
an increase in both cholesterol and EPA supply can independently increase growth. Assuming strictly essential resources according to Liebig's law would lead to an overestimation of growth rates by up to 
$0.07 \mathrm{~d}^{-1}$ (i.e. $13 \%$ of the overall growth response) compared to the assumption of interactive-essential resources according to the MLH (Fig. 4). This overestimation was also observed in a similar magnitude (up to $0.06 \mathrm{~d}^{-1}$, see Appendix S3) when differences in predicted growth rates were calculated using the estimated parameters of both the Bertalanffy model based on the MLH (Table 2, model VI) and the Bertalanffy model based on Liebig's law (Table 2, model II).

\section{DISCUSSION}

The objective of this study was to test the suitability of existing multiple resource limitation models to describe experimentally determined nutrient-limited growth responses of a herbivorous consumer. We show that the growth of the herbivore D. magna in a system with a diet of two potentially limiting nutrients is constrained simultaneously by both nutrients within a certain range of nutrient availabilities and that the growth rate is better described by the concept of the MLH than by the concept of Liebig's law of the minimum. This indicates that Liebig's minimum rule is too simple to accurately describe co-limited growth responses of consumers across a full range of nutrient availabilities.

An obvious drawback of Liebig's minimum rule is the inherent abrupt switch between limiting nutrients, which appears to be rather unlikely and oversimplified. Thus, more realistic descriptions of smooth transitions between nutrient limitations are required, which allow to assess interactive effects between limiting nutrients. The MLH based on interactive-essential resources considers such smooth transitions and, in our study, described the growth of $D$. magna better than Liebig's law based on strictly essential resources (judged by the obtained differences in $N L L$-values). The differences in goodness of fit measures obtained for the two concepts of co-limitation appear relatively small, probably because the range of resource availabilities at which the model outcomes substantially differ, i.e. at co-limiting conditions, is rather small (case $[\mathrm{C}]$ in. Figs $3 \mathrm{~d}$ and 4 , Appendix S3). In the remaining nutrient space, in which growth was limited primarily by a single nutrient, growth rates predicted by the models based on the MLH and Liebig's law are similar, suggesting that Liebig's law of the minimum can be applied appropriately when growth is predominantly limited by only one nutrient, but not when multiple nutrients approach potentially co-limiting concentrations.

Based only on model comparisons, it is difficult to decide which concept of co-limitation is more suitable to describe growth. Therefore, we also compared selected growth response data within the different limitation scenarios identified actoss the nutrient space

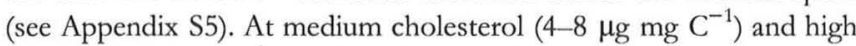
EPA $\left(>3 \mu \mathrm{g} \mathrm{m} \mathrm{C}^{-1}\right.$ ) availabilities, growth rates increased only with cholesterol supplementation, whereas at low EPA $\left(<0.5 \mu \mathrm{g} \mathrm{mg} \mathrm{C}^{-1}\right)$ and high cholesterol $\left(>11 \mu \mathrm{g} \mathrm{mg} \mathrm{C} \mathrm{C}^{-1}\right)$ availabilities growth rates increased only with EPA supplementation. At medium cholesterol and low EPA availabilities, however, growth rates increased both upon cholesterol and upon EPA supplementation (see Appendix S5), which indicates a simultaneous limitation by both nutrients in this range of resource availabilities and thus, supports the applicability of the MLH at co-limiting conditions.

In another study, different co-limitation models based on Monod kinetics were compared by applying nonlinear least-squares regressions to 11 data sets of nutrient-limited autotrophs (O'Neill et al. 1989). In about half of the investigated data sets, the Monod model based on Liebig's law showed a better goodness of fit measure compared to a model which is equivalent to our applied Monod model based on the MLH (cf. Liebig and Baule model in O'Neill et al. 1989). The authors noted, however, that the Liebig model was worse than other models considering interactions between limiting nutrients because it often converged to very unrealistic parameter values and performed better only for the scantiest data sets. For an appropriate differentiation between co-limitation models comprehensive data sets are required, which provide sufficient data over a large range of nutrient availabilities (O'Neill et al. 1989). Our study provides the first of such data sets for growth responses of a consumer. One of the best performing models in O'Neill et al. (1989) was their PAT model, which in our study also resulted in a better goodness of fit than the Monod model based on either Liebig's law or the MLH (Table 2, cf. model I, III, IV). Thus, when using Monod kinetics to describe growth responses, interactions between co-limiting nutrients might be better described by the PAT model than by the product of Monod functions. However, the comparisons of our nonlinear regression results strongly indicate that the underlying growth responses follow Bertalanffy functions rather than hyperbolic functions (i.e. the Monod, PAT and SU models), showing that the growth of Daphnia in this study can be better described by the Bertalanffy growth models, which, for instance, better approached the actual maximum growth rates than the other models (cf. $g_{\max }$ in Table 2).

As hypothesised (Fig. 2), the largest differences between the two contrasting concepts of co-limitation were found at strongly colimiting conditions (Fig. 4, see Appendix S3). In this range of resource availabilities, the calculated differences in growth rates indicated that the co-limited growth of $D$. magna was overestimated by applying Liebig's law as compared to the MLH (Fig. 4, see Appendix S3). The maximum values of differences in predicted growth rates were moderate (11-13\% of the overall growth response, depending on the calculation method; see Appendix S3). However, recent work demonstrated that details in nutrient uptake kinetics are qualitatively and quantitatively important for the outcome of population and community models (Fussmann \& Blasius 2005; Poggiale et al. 2010). More specifically, the implementation of different multi-nutrient uptake functions to describe nutrient acquisition of phytoplankton had significant effects on zooplankton-phytoplankton interactions and thus for population dynamics (Poggiale et al. 2010). Likewise, in a simple predator-prey model, nearly indistinguishable resource uptake functions led to strong changes in community dynamics and stability patterns (Fussmann \& Blasius 2005). These examples indicate that applying the most suitable co-limitation concept is very important to accurately predict primary and secondary production and associated food web processes.

Here, we classified cholesterol and EPA as interactive-essential resources according to the MLH rather than strictly essential resources according to Liebig's law. Although the range of resource concentrations at which interactive effects between the co-limiting nutrients were observed was rather small (Fig. 4, 1-11 $\mu \mathrm{g}$ cholesterol $\mathrm{mgC}^{-1}$, $<1.5 \mu \mathrm{g}$ EPA mg C ${ }^{-1}$ ), Daphnia may encounter such resource supplies in the field. (1) In pelagic freshwater habitats, in particular in cyanobacteria-dominated lakes and ponds, EPA concentrations within the co-limiting range $\left(<1.5 \mu \mathrm{g} \mathrm{EPA} \mathrm{mg} \mathrm{C}^{-1}\right)$ were frequently reported (Müller-Navarra et al. 2004; Sperfeld \& Wacker 2011). (2) Sterol concentrations in lakes and ponds which are available for Daphnia have not been determined yet. However, dietary sterol concentrations in the range of co-limiting conditions are also most likely to occur when cyanobacteria dominate the phytoplankton 
because cyanobacteria lack not only highly unsaturated fatty acids such as EPA but also sterols (Volkman 2003). Laboratory experiments using food mixtures with cyanobacteria and algae containing saturating amounts of sterols revealed that more than $50 \%$ cyanobacteria in the food (i.e. $<5.6 \mu \mathrm{g}$ sterol $\mathrm{mg} \mathrm{C}^{-1}$ ) can result in sterollimited growth of Daphnia (Martin-Creuzburg et al. 2005, 2009), a situation frequently occurring in nature.

Recently it has been shown in laboratory experiments that dietary $\mathrm{P}$ and cholesterol can co-limit the growth of D. magna thereby affecting the $\mathrm{P}$ homeostasis of the animal in an interactive manner (Lukas et al. 2011). Thus, the concept of co-limiting nutrients should be incorporated explicitly also in stoichiometric models dealing with single elements which have been shown already to result in qualitatively different predictions of population dynamics compared to solely food quantity based models (Andersen $e t$ al. 2004). The effect of co-limiting conditions on consumers' stoichiometry may have consequences not only for individual growth and population dynamics but also for nutrient release and recycling as element deficiencies in consumers will reduce overall recycling rates and may change the proportions between recycled elements (Andersen et al. 2004; Anderson et al. 2005).

A major challenge for future research will be to identify the mechanisms responsible for interactive effects between co-limiting nutrients, which can substantially affect mass-specific as well as population growth rates (Martin-Creuzburg et al. 2010; Lukas et al. 2011). Adaptive strategies based on optimal foraging theory could explain such interactive effects (Tilman 1987); more energy can be allocated to acquire a more limiting resource if other resources are sufficiently available. Mechanisms such as compensatory feeding or selection of complementary food sources, as observed for terrestrial herbivores (e.g. Simpson et al. 2004), cannot be applied to rather unsclective filter-feeders such as Daphnia. In contrast, daphnids may respond to stoichiometrically imbalanced diets after ingestion (Darchambeau et al. 2003), e.g. by down-regulating the assimilation of nutrients in excess and up-regulating the assimilation of limiting nutrients (Hessen \& Anderson 2008). An alternative explanation for interactive effects between co-limiting nutrients that has to be tested experimentally could be that the ability to assimilate or transport a limiting nutrient within the body is constrained when the animal is stressed by the limitation of other essential nutrients. Accordingly, the efficiency in utilising a specific nutrient would be maximised when the animal is not limited by other nutrients simultaneously.

A more mechanistic explanation for interactive effects of multiple nutrients could be derived from the SU concept (Kooijman 1998). In consumers, a SU can be considered as a biomass production unit, in which the production rate depends on the supply fluxes of essential nutrients (Muller et al. 2001). With decreasing supply flux of an essential nutrient the probability of this nutrient arriving at the SU declines, resulting in decreased biomass production. A decrease in the supply flux of an additional nutrient will intensify the decrease in biomass production, which consequently leads to smooth transitions between limiting nutrients and thus, to the observed interactive effects. In homogeneous environments, supply fluxes of essential nutrients might be proportional to their concentrations and thus, supply fluxes can be substituted by concentrations (Muller et al. 2001). We applied a SU model based on nutrient concentrations to our data as $D$. magna experienced a well mixed resource environment in our experiment. The SU model described our data equally well as the aforementioned PAT model from O'Neill et al. (1989). The SU and PAT model assume nutrient supplies according to a Poisson distribution and resulted in better fits than our Monod model based on the MLH (cf. Table 2, model III, IV, V). This suggests that modelling nutrient arrival according to a Poisson process is indeed a promising method to describe interactions between limiting nutrients (O’Neill et al. 1989; Kooijman 1998).

The SU, PAT or Bertalanffy model based on the MLH can be incorporated as resource-based production rate functions into more sophisticated models describing consumer's growth and physiology, such as dynamic energy budget (DEB) models (Kooijman 2000). DEB models comprise both reserve and structural compartments of the animal and thus consider the storage of nutrients in the simulation of biomass production. This might be important in particular for essential fatty acids, as they can be stored by animals in rather large amounts (Wacker \& Martin-Creuzburg 2007).

We show here that the growth of our model herbivore $D$. magna can be limited by two essential nutrients in an interactive manner, which challenges the use of Liebig's minimum rule to describe nutrientlimited growth of herbivorous consumers. Hence, to accurately predict demographic changes and population dynamics, models are required which consider different types of co-limitations and potential interactive effects among limiting nutrients. We hypothesise that colimitation is a common phenomenon in nature not only for plants, but also for herbivorous consumers, as they are particularly sensitive to constraints in food quality.

\section{ACKNOWLEDGEMENTS}

We thank Silvia Heim and Sabine Donath for technical assistance, Matthijs Vos, Ursula Gaedke and Gregor Fussman for valuable comments on an earlier draft of this manuscript and Adisel Montana for improving the language. The manuscript profited strongly from reviews by John Pastor and two anonymous referees. This study was supported by the German Research Foundation (DFG, WA 2445/3 1\& WA 2445/4-1)

\section{AUTHORSHIP}

ES performed the experiments and wrote the first draft of the manuscript. All authors analysed the data and contributed substantially to revisions of the manuscript.

\section{REFERENCES}

Allgeier, J.E., Rosemond, A.D. \& Layman, C.A. (2011). The frequency and magnitude of non-additive responses to multiple nutrient enrichment. J. Appl. Ecol, $48,96-101$.

Andersen, T., Elser, J.J. \& Hessen, D.O. (2004). Stoichiometry and population dynamics. Ecol. Lett., 7, 884-900.

Anderson, T.R., Boersma, M. \& Raubenheimer, D. (2004). Stoichiometry: linking elements to biochemicals. Ecology, 85, 1193-1202.

Anderson, T.R., Hessen, D.O., Elser, J.J. \& Urabe, J. (2005). Metabolic stoichiometry and the fate of excess carbon and nutrients in consumers. Am. Nat., 165 $1-15$.

Arrigo, K.R. (2005). Marine microorganisms and global nutrient cycles. Nature, 437, 349-355.

Baule, B. (1918). Zu Mitscherlichs Gesetz der physiologischen Beziehungen. Landwirtschaftliche Jabrbiicber, 51, 363-385.

Behmer, S.T. \& Nes, W.D. (2003). Insect sterol nutrition and physiology: a global overview. Adv. Insect Physiol., 31, 1-72.

Darchambeau, F., Faerøvig, P.J. \& Hessen, D.O. (2003). How Daphnia copes with excess carbon in its food. Oecologia, 136, 336-346. 
Davidson, E.A. \& Howarth, R.W. (2007). Environmental science: nutrients in synergy. Nature, 449, 1000-1001.

Elser, J.J., Fagan, W.F., Denno, R.F., Dobberfuhl, D.R., Folarin, A., Huberty, A. et al. (2000). Nutritional constraints in terrestrial and freshwater food webs. Nature, 408, 578-580.

Elser, J.J., Bracken, M.E.S., Cleland, E.E., Gruner, D.S., Harpole, W.S., Hillebrand, H. et al. (2007). Global analysis of nitrogen and phosphorus limitation of primary producers in freshwater, marine and terrestrial ecosystems. Ecol. Lett., 10, 11351142.

Fussmann, G.F. \& Blasius, B. (2005). Community response to enrichment is highly sensitive to model structure. Biol. Lett., 1, 9-12.

Gleeson, S.K. \& Tilman, D. (1992). Plant allocation and the multiple limitation hypothesis. Am. Nat., 139, 1322-1343.

Guillard, R.R. (1975). Cultures of phytoplankton for feeding of marine invertebrates. In: Culture of marine invertebrate animals (eds Smith, W.L. \& Chanley, M.H.) Plenum Press, New York, pp. 26-60.

Harpole, W.S., Ngai, J.T., Cleland, E.E., Seabloom, E.W., Borer, E.T., Bracken, M.E.S. et al. (2011). Nutrient co-limitation of primary producer communities. Ecol. Lett., 14, 852-862.

Hessen, D.O. \& Anderson, T.R. (2008). Excess carbon in aquatic organisms and ecosystems: physiological, ecological, and evolutionary implications. Limnol. Oceanogr., 53, 1685-1696.

Holling, C.S. (1959). The components of predation as revealed by a study of smallmammal predation of the European pine sawfly. Can. Entomol, 91, 293-320.

Keating, K.I. (1985). The influence of vitamin $B_{12}$ deficiency on the reproduction of Daphnia pulex Leydig (Cladocera). J. Crustacean Biol., 5, 130-136.

Kooijman, S.A.L.M. (1998). The synthesizing unit as model for the stoichiometric fusion and branching of metabolic fluxes. Biophys. Chem., 73, 179-188.

Kooijman, S.A.L.M. (2000). Dynamic Energy and Mass Budgets in Biological Systems, 2nd edn. Cambridge University Press, Cambridge, UK.

Lukas, M., Sperfeld, E. \& Wacker, A. (2011). Growth rate hypothesis does not apply across colimiting conditions: cholesterol limitation affects phosphorus homoeostasis of an aquatic herbivore. Funct. Ecol, 25, 1206-1214.

Martin-Creuzburg, D. \& Von Elert, E. (2009). Ecological significance of sterols in aquatic food webs. In: Lipids in Aquatic Ecosystems (eds Arts, M.T., Brett, M.T. \& Kainz, M.). Springer, New York, pp. 43-64.

Martin-Creuzburg, D., Wacker, A. \& Von Elert, E. (2005). Life history consequences of sterol availability in the aquatic keystone species Daphnia. Oecologia, 144, 362-372.

Martin-Creuzburg, D., Von Elert, E. \& Hoffmann, K.H. (2008). Nutritional constraints at the cyanobacteria-Daphnia magna interface: the role of sterols. Limnol. Oceanogr, 53, 456-468.

Martin-Creuzburg, D., Sperfeld, E. \& Wacker, A. (2009). Colimitation of a freshwater herbivore by sterols and polyunsaturated fatty acids. Proc. R. Soc. Lond. Ser. B-Biol. Sci., 276, 1805-1814.

Martin-Creuzburg, D., Wacker, A. \& Basen, T. (2010). Interactions between limiting nutrients and associated consequences for growth and reproduction of Daphnia magna. Limnol. Oceanogr, 55, 2597-2607.

Monod, J. (1950). La technique de culture continue. Theorie et application. Annales de / Institut Pasteur; 79, 390-410.

Motulsky, H.J. \& Cristopoulus, A. (2004). Fitting Models to Biological Data Using Linear and Nonlinear Regression. A Practical Guide to Curve Fitting. Oxford University Press, Oxford.

Muller, E.B., Nisbet, R.M., Kooijman, S.A.L.M., Elser, J.J. \& McCauley, E. (2001). Stoichiometric food quality and herbivore dynamics. Ecol. Lett., 4, 519-529.

Müller-Navarra, D.C., Brett, M.T., Liston, A.M. \& Goldman, C.R. (2000). A highly unsaturated fatty acid predicts carbon transfer between primary producers and consumers. Nature, 403, 74-77.

Müller-Navarra, D.C., Brett, M.T., Park, S., Chandra, S., Ballantyne, A.P., Zorita, E. et al. (2004). Unsaturated fatty acid content in seston and tropho-dynamic coupling in lakes. Nature, 427, 69-72.

O’Neill, R.V., Deangelis, D.L., Pastor, J.J., Jackson, B.J. \& Post, W.M. (1989). Multiple nutrient limitations in ecological models. Ecol. Model., 46, 147-163.

Parrish, C.C. (2009). Essential fatty acids in aquatic food webs. In: Lipids in Aquatic Ecosystems (eds Arts, M.T., Brett, M.T. \& Kainz, M.). Springer, New York, pp. 309-326.
Poggiale, J.C., Baklouti, M., Queguiner, B. \& Kooijman, S.A.L.M. (2010). How far details are important in ecosystem modelling: the case of multi-limiting nutrients in phytoplankton-zooplankton interactions. Pbil. Trans. R. Soc. B-Biol. Sci., 365 3495-3507.

Raubenheimer, D. \& Simpson, S.J. (2004). Organismal stoichiometry: quantifying non-independence among food components. Ecology, 85, 1203-1216.

Saito, M.A., Goepfert, T.J. \& Ritt, J.T. (2008). Some thoughts on the concept of colimitation: three definitions and the importance of bioavailability. Limnol Oceanogr., 53, 276-290.

Simpson, S.J., Sibly, R.M., Lee, K.P., Behmer, S.T. \& Raubenheimer, D. (2004) Optimal foraging when regulating intake of multiple nutrients. Anim. Behav., 68, 1299-1311.

Sperfeld, E. \& Wacker, A. (2011). Temperature- and cholesterol-induced changes in eicosapentaenoic acid limitation of Daphnia magna determined by a promising method to estimate growth saturation thresholds. Limnol. Oceanogr., 56, 1273-1284.

Stanley-Samuelson, D.W., Jurenka, R.A., Cripps, C., Blomquist, G.J. \& Derenobales, M. (1988). Fatty acids in insects: composition, metabolism, and biological significance. Arch. Insect Biochem. Physiol., 9, 1-33.

Sterner, R.W. \& Elser, J.J. (2002). Ecological Stoichiometry: The Biology of Elements From Molecules to the Biosphere. Princeton University Press, Princeton, NJ.

Tilman, D. (1982). Resource Competition and Community Structure. University Press, Princeton, NJ.

Tilman, D. (1987). Further thoughts on competition for essential resources. Theor: Popul. Biol., 32, 442-446.

Volkman, J.K. (2003). Sterols in microorganisms. Appl. Microbiol. Biotechnol., 60, 495506.

Von Bertalanffy, L. (1957). Quantitative laws in metabolism and growth. $Q$. Rev. Biol., 32, 217-231.

Von Liebig, J. (1840). Die Organische Chemie in ihre Anwendung auf Agricultur und Physiologie. F. Vieweg und Sohn, Braunschweig.

Wacker, A. \& Martin-Creuzburg, D. (2007). Allocation of essential lipids in Daphnia magna during exposure to poor food quality. Funct. Ecol, 21, 738-747.

Wacker, A. \& Von Elert, E. (2001). Polyunsaturated fatty acids: evidence for nonsubstitutable biochemical resources in Daphnia galeata. Ecology, 82, 2507-2520.

\section{SUPPORTING INFORMATION}

Additional Supporting Information may be found in the online version of this article:

Appendix S1 Carbon-based cholesterol and eicosapentaenoic acid (EPA) concentrations in the experimental food treatments.

Appendix S2 Additional co-limitation models and fitting results.

Appendix S3 Estimation and interpretation of differences in predicted growth rates between the two proposed concepts of co-limitation obtained by two different calculation methods.

Appendix S4 Mass-specific growth rates of Daphnia magna in all experimental food treatments.

Appendix S5 Examples of (co-)limitation scenarios in the twodimensional nutrient space. 